\title{
Inverse Primakoff Scattering as a Probe of Solar Axions at Liquid Xenon Direct Detection Experiments
}

\author{
James B. Dent $\odot,{ }^{1, *}$ Bhaskar Dutta, ${ }^{2, \dagger}$ Jayden L. Newstead $\oplus^{3, *}$ and Adrian Thompson $\oplus^{2, \S}$ \\ ${ }^{1}$ Department of Physics, Sam Houston State University, Huntsville, Texas 77341, USA \\ ${ }^{2}$ Mitchell Institute for Fundamental Physics and Astronomy, Department of Physics and Astronomy, Texas A\&M University, \\ College Station, Texas 77843, USA \\ ${ }^{3}$ ARC Centre of Excellence for Dark Matter Particle Physics, School of Physics, The University of Melbourne, \\ Victoria 3010, Australia
}

(Received 29 June 2020; revised 31 July 2020; accepted 19 August 2020; published 24 September 2020)

\begin{abstract}
We show that XENON1T and future liquid xenon (LXe) direct detection experiments are sensitive to axions through the standard $g_{a \gamma} a F \tilde{F}$ operators due to inverse-Primakoff scattering. This previously neglected channel significantly improves the sensitivity to the axion-photon coupling, with a reach extending to $g_{a \gamma} \sim 10^{-10} \mathrm{GeV}^{-1}$ for axion masses up to a $\mathrm{keV}$, thereby extending into the region of heavier QCD axion models. This result modifies the couplings required to explain the XENON1T excess in terms of solar axions, opening a large region of $g_{a \gamma}-m_{a}$ parameter space that is not ruled out by the CAST helioscope experiment and reducing the tension with the astrophysical constraints. We explore the sensitivity to solar axions for future generations of LXe detectors that can exceed future helioscope experiments, such as IAXO, for a large region of parameter space.
\end{abstract}

DOI: 10.1103/PhysRevLett.125.131805

Dark matter direct detection experiments, initially designed to search for WIMP-like dark matter, have been adapted more broadly as detectors of beyond standard model physics. Notable among the wide class of beyond standard model physics searches at direct detection facilities is the extraordinary sensitivity to possible axion or axionlike particles [we will use the generic "axion" (and symbol a) to encompass both cases, with the modifier QCD added when dealing with specific models developed to address the strong $C P$ problem of $\mathrm{QCD}]$ coupling to standard model particles (SM) [1-7]. By examining electronic recoils produced by a solar axion flux through the detector, these searches have probed a variety of $a$-SM couplings including axion-electron, axion-photon, and axion-nucleon interactions.

Recently, the XENON1T collaboration announced an observed excess of electron recoils in their low energy (1-30 keV) data, with a rise above the background-only model occurring below $7 \mathrm{keV}$ [8]. The solar axion flux is predicted to reside mostly in this energy range, making it a well-motivated hypothesis for the excess. The collaboration showed that a solar axion model can fit the data with a $3.5 \sigma$

Published by the American Physical Society under the terms of the Creative Commons Attribution 4.0 International license. Further distribution of this work must maintain attribution to the author(s) and the published article's title, journal citation, and DOI. Funded by SCOAP ${ }^{3}$. significance, which is reduced to $2.1 \sigma$ if an unconstrained tritium background is introduced in the fitting.

XENON1T placed constraints in a three-dimensional confidence limit volume in the parameter space of the axion-electron coupling, $g_{a e}$, along with the products $g_{a e} g_{a \gamma}$ and $g_{a e} g_{\mathrm{an}}^{\text {eff }}$, where $g_{a \gamma}$ and $g_{\mathrm{an}}^{\text {eff }}$ characterize the strength of axions coupling to photons and nucleons, respectively. These constraints were shown to be competitive with (or exceeding in some regions) the axion helioscope experiment CAST [9] and the xenon-based dark matter direct detection experiments LUX [1] and PandaX-II [2]. The preferred region for the solar axion interpretation of the XENON1T result is in severe tension with astrophysical bounds, as discussed in [10,11] (see [12] for a recent review that includes updated astrophysical bounds).

The analysis calculated the expected event rates produced by a solar axion flux consisting of three components arising from each of the couplings mentioned above. The XENON1T analysis considered detection through axioelectric scattering, the axion-analog of the photoelectric effect, off Xe electrons. This scattering process depends only on the $g_{a e}$ axion coupling, and not on either $g_{a \gamma}$ or $g_{\text {an }}^{\text {eff }}$.

There is an alternative means of producing electron recoils through axion scattering that does not rely on the $g_{a e}$ coupling-namely through Primakoff scattering. In Primakoff scattering (also called the inverse Primakoff effect) where an incident axion scatters off a charged particle through the $g_{a \gamma}$ coupling, producing an outgoing photon and recoil of the target particle (see the Supplemental Material [13] for a diagram). This channel 
occurs through a coherent interaction with the entire atomic form factor, not to be confused with a similar process involving the coherent interaction with external electromagnetic fields. The inverse Primakoff scattering process has been considered in several works [14-19], including a recent analysis of the sensitivity of reactor neutrino experiments to axionlike particles with low-threshold detectors [20].

In this Letter, we first describe the inverse Primakoff channel for axion detection in XENON1T (which can be applied to any direct detection experiments). We then explore the axion model parameter space for regions which can fit the XENON1T excess through Primakoff scattering within the detector. We demonstrate that the current XENON1T excess can be well fit purely through a $g_{a \gamma}$ coupling (for both solar production and experimental detection). We show that there are regions of coupling and axion mass parameter space which fit the excess and are not ruled out by the CAST experiment. This region has constraints from HB stars which, however, can be evaded in the context of particle physics models [21-27]. If this excess is instead due to an unmodeled background, we show the constraint emerging from this inverse Primakoff channel at the ongoing and future detectors.

Models.-Peccei and Quinn introduced a new global chiral symmetry into the $\mathrm{SM}$ in order to solve the strong $C P$ problem [28]. This symmetry is broken dynamically and the resulting pseudo Nambu-Goldstone boson is the axion $[29,30]$. Although axion couplings to all SM particles can be considered, in the present work we examine the couplings to photons and leptons within the interaction Lagrangian

$$
\mathcal{L} \supset-\frac{1}{4} g_{a \gamma} a F_{\mu \nu} \tilde{F}^{\mu \nu}+i g_{a e} a \bar{\psi} \gamma \gamma^{5} \psi .
$$

These couplings are model dependent, with some models allowing lepton couplings only beginning at one-loop order. Within a given model, these couplings are not strictly independent, as loop effects can correlate them. However, in the present work we allow these parameters to be independently fit to the data. We will also comment on the common QCD axion models of Dine, Fischler, Srednicki, and Zhitnitskii (DFSZ) [31,32], and Kim, Shifman, Vainshtein, and Zakharaov (KSVZ) [33,34], which provide specific, model dependent forms for $g_{a \gamma}$ and $g_{a e}$. In addition, effective couplings to nucleons (of a similar form as the electron interaction term [35]) will be included in the next section.

The explicit coupling correlations can be made to connect our phenomenological axion model to specific DFSZ and KSVZ QCD axion models in the analysis. For these we make use of the coupling relationships which can be found in refs. [36-39]. The KSVZ- and DFSZ-type axion models can be classified by the ratio anomaly parameters $E$ and $N$ specific to each variety of model.
In addition, the definitions of $g_{a \gamma}$ and $g_{a e}$ are correlated in each model ( $g_{a e}$ being loop induced in KSVZ models, while $g_{a \gamma}$ is loop induced in DFSZ), and depend on the QCD scale factor $\Lambda \sim 1 \mathrm{GeV}$ and mixing parameter $\tan \beta$ (just for DFSZ models). We assume $\tan \beta=140$ (DFSZ I) and $\tan \beta=0.28$ (DFSZ II), taken from fits to accommodate unitarity and stellar cooling [39]. Although our analysis will permit $g_{a \gamma}$ and $g_{a e}$ to be free, we can use their correlations in the varieties of KSVZ and DFSZ models to pick out subspaces of the total parameter space. Various instances of these models have been explored over a range of $E / N$ values [40].

Analysis.-We consider two possibilities of solar axion production (for details regarding solar axion fluxes, see Refs. [35,41-46] and the Supplemental Material [13]) and subsequent scattering within the XENON1T volume. First, we examine Primakoff production in the sun, followed by photon production through Primakoff scattering in the detector (this combination is purely dependent on $g_{a \gamma}$ ). We note here that solely using this coupling approximates the phenomenology of $g_{a \gamma}$ dominated axion models, e.g., KSVZ-type axion models, for which axion-electron interactions happen through loop-induced processes, and would be suppressed. This also allows a direct comparison with other experiments, such as haloscopes and helioscopes, which have sensitivity to $g_{a \gamma}$ as a function of the axion mass, $m_{a}$. Second, we analyze a nonzero $g_{a e}$ in conjunction with a nonzero $g_{a \gamma}$, which allows for both $\mathrm{ABC}$ and Primakoff solar production, along with axioelectric and Primakoff scattering detection. This analysis is complementary to that of XENON1T, which allowed for the possibility of Primakoff production, but only detection through the axion-electron scattering controlled by the $g_{a e}$ coupling.

Inverse Primakoff scattering allows solar axions to coherently scatter from the Xe atomic electric field and backconvert into photons in the detector volume; $a Z \rightarrow \gamma Z$, proceeding through a $t$-channel photon exchange. Therefore the inverse Primakoff scattering contributes to the axion hypothesis and must be included. The final state photon will have a short mean free path $(\sim \mu \mathrm{m})$ and cause an electronlike recoil in the LXe TPC. This is verified by XENON1T's ${ }^{37} \mathrm{Ar}$ calibration; ${ }^{37} \mathrm{Ar}$ decays via electron capture of a $K$-shell electron to ${ }^{37} \mathrm{Cl}$, the core hole is filled from an outer shell electron, which emits a $2.8 \mathrm{keV}$ photon with $90 \%$ branching fraction $[8,47]$. Additionally, the xenon response to low energy photons was measured by the LUX collaboration who found that, in the region of interest, the response is approximately the same as for electron recoils [48]. Therefore it is reasonable to assume that all inverse Primakoff events are detected with the same efficiency and energy resolution as electron recoils.

For an axion of momentum $k_{a}$, the inverse Primakoff cross section is given by $[14,15,17]$ 


$$
\sigma\left(k_{a}\right)=\frac{Z^{2} \alpha g_{a \gamma}^{2}}{2}\left(\frac{2 r_{0}^{2} k_{a}^{2}+1}{4 r_{0}^{2} k_{a}^{2}} \ln \left(1+4 r_{0}^{2} k_{a}^{2}\right)-1\right)
$$

where $r_{0}$ is the screening length for which we take as the Wigner-Seitz radius in LXe, $2.45 \AA$.

We consider inverse Primakoff scattering in addition to the axioelectric absorption process outlined in the analysis performed by XENON1T (see also [35,49-51]). Also, it is possible that axions undergo inverse Compton scattering off electrons at rest in LXe, $a e^{-} \rightarrow \gamma e^{-}$[14], but this is a subdominant process $(\propto Z)$ in comparison to axioelectric scattering $\left(\propto Z^{5}\right)$. If both axion-photon and axion-electron couplings are present, there are interference terms present in the total matrix element of the combined processes, which are also subdominant, but we include them as a matter of completeness.

To predict the event spectra from axions produced through $\mathrm{ABC}$, Primakoff, and ${ }^{57} \mathrm{Fe}$, we convolve the fluxes in each case with the total cross sections, for inverse Primakoff scattering or axioelectric absorption, and multiply by the detector efficiency [8]. In addition, we approximate the detector response for the energy resolution by convolving the simulated differential event distribution with an energy-dependent Gaussian smearing function $[8,52,53]$. The event distribution for Primakoff-produced axions that undergo inverse Primakoff scattering in the LXe fiducial volume over a ton-year exposure is shown in Fig. 1.

We perform a likelihood analysis given the data and " $B_{0}$ " background hypothesis taken in [8] and our axion signal hypotheses using the Bayesian inference package multinest [54-56]. A binned log-Gaussian likelihood is constructed over bins $i$, with signal event rates $\left\{\mu_{i}\right\}$ and observed events $\left\{n_{i}\right\}$ ranging from 1 to $29 \mathrm{keV}$, taken with errors $\sigma_{i}$ reported by XENON1T.

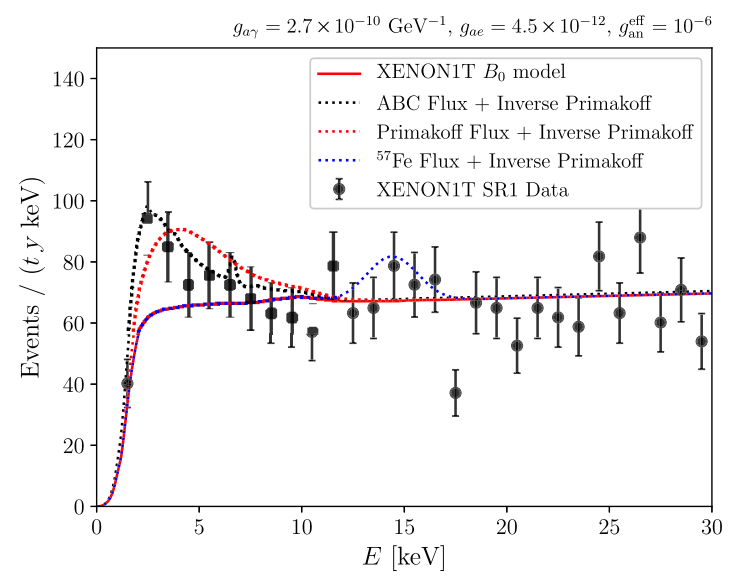

FIG. 1. The event rate distributions for inverse Primakoff scattering in LXe for a ton-year exposure from Primakoffproduced, ABC-produced, and ${ }^{57} \mathrm{Fe}$-produced axions are shown for select choices of axion couplings, and added to the " $B_{0}$ " background model.
We wish to investigate several scenarios of signal and background models in the context of the excess, enumerated as follows: (i) Primakoff-produced axions detected through solely inverse Primakoff scattering, (ii) Primakoffproduced and scattered axions with an additional ${ }^{3} \mathrm{H}$ component, (iii) the ${ }^{3} \mathrm{H}$ component alone, repeating the methods used in the XENON1T analysis, (iv) all production mechanisms (ABC, Primakoff, ${ }^{57} \mathrm{Fe}$ ) and all scattering channels (Primakoff, axioelectric, Compton) allowed in the detector, and, finally, (v) all flux components and scattering channels along with an unconstrained ${ }^{3} \mathrm{H}$ component. Fits (i) and (ii) aim to test the robustness of the Primakoff-only (pure- $g_{a \gamma}$ ) fit after introducing a ${ }^{3} \mathrm{H}$ background component, while (iii) validates the ${ }^{3} \mathrm{H}$-only fit. Fits (iv) and (v) aim to test the same robustness when all axion production and detection mechanisms are allowed. For each of these cases we will assume flat priors over appropriately large intervals on the free parameters $\left(g_{a e}, g_{a \gamma}\right.$, and $\left.g_{\mathrm{an}}^{\text {eff }}\right)$ in the likelihood scan. We keep the axion mass fixed below $100 \mathrm{eV}$, since the production and scattering rates remain unchanged in this limit, and keep $g_{a \gamma}$ and $g_{a e}$ sufficiently small as to avoid $a \rightarrow \gamma \gamma$ decays that would be ruled out several constraints.

We will also consider alternative scenarios where the low energy excess either disappears with more exposure at third-generation xenon experiments, or that the background model becomes more well understood and shows no excess. We can simulate these possibilities to forecast future exclusions in parameter space. Future limits and the five cases that we consider for the analysis of the excess are discussed in the next section.

Fit results.-After checking all five cases described in the previous section with the likelihood-ratio test statistic, we find that the ${ }^{3} \mathrm{H}$ unconstrained model rejects the background-only hypothesis at a $2.3 \sigma$ level, in agreement with the XENON1T result. When Primakoff production and detection mechanisms are added to the signal model that includes the unconstrained ${ }^{3} \mathrm{H}$ component, we find a significance of $2.6 \sigma$, while if we remove the ${ }^{3} \mathrm{H}$ component and just include Primakoff production and detection, we reject the background-only hypothesis at $3.1 \sigma$, slightly less significant than the XENON1T result which omitted the inverse Primakoff detection component. This may be intuitively understood by the shape difference between the Primakoff flux with inverse Primakoff response, shown as the red dotted curve in Fig. 1, and the response from the ABC-produced axioelectric absorption which is peaked at lower energies more than the inverse Primakoff response. Finally, if we allow for all fluxes and detection channels that we considered to be present in the likelihood scan, we find a rejection of the background at a level of $3.7 \sigma$, mildly higher than the XENON1T result, while if we also include an unconstrained ${ }^{3} \mathrm{H}$, the significance is reduced to $2.95 \sigma$.

For the purely Primakoff-driven production and detection scenario, in Fig. 2 we display our best fit region in the $g_{a \gamma}-m_{a}$ parameter space for the XENON1T excess, as well 


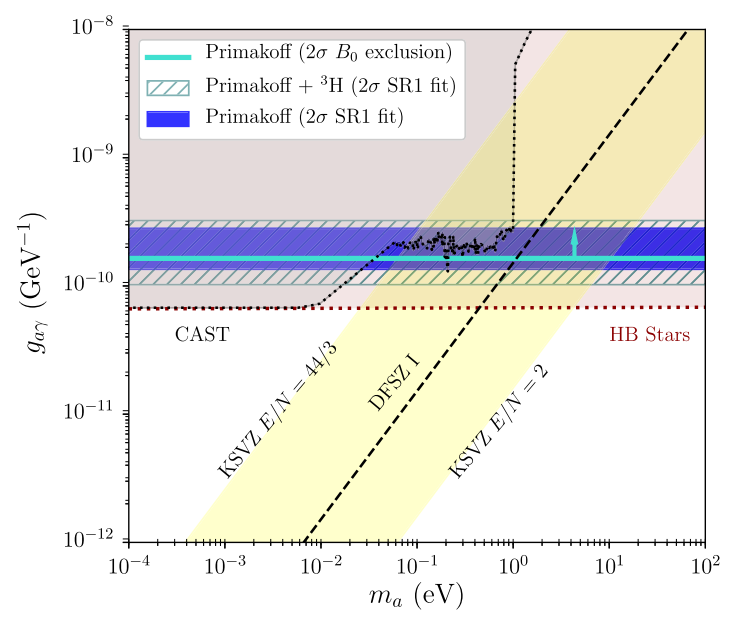

FIG. 2. The $2 \sigma$ credible band for Primakoff-produced solar axions undergoing solely inverse-Primakoff scattering in XENON1T (blue band) is compared against the $2 \sigma$ limit when ${ }^{3} \mathrm{H}$ is included as a background as well as the signal hypothesis (hatched band). We also show the $2 \sigma$ bound from the Primakoff signal hypothesis tested against the $B_{0}$ background, simulating a no-excess scenario. We discuss the existing constraints in the text.

as the current limits from the CAST helioscope and astrophysical bounds. The CAST limits [57] provide a bound of $g_{a \gamma}<0.66 \times 10^{-10} \mathrm{GeV}^{-1}$ (95\% C.L.) for $m_{a}<0.02 \mathrm{eV}$, and $g_{a \gamma}<2 \times 10^{-10} \mathrm{GeV}^{-1}$ (95\% C.L.) for $m_{a}<0.7 \mathrm{eV}$. The excess explanation evades the CAST constraint for $m_{a}>0.03 \mathrm{eV}$. Not pictured are the other constraints of $g_{a \gamma}<4.1 \times 10^{-10} \mathrm{GeV}^{-1}$, from a combined global analysis of helioseismology and solar neutrino arguments [58], and $g_{a \gamma} \lesssim 6 \times 10^{-10} \mathrm{GeV}^{-1}$ for $0.8 \lesssim m_{a} \lesssim 1.0 \mathrm{eV}$, from SUMICO [59], both of which are evaded by the XENON1T fit for all masses considered.

Bounds from the $R$ parameter-the ratio between the number of horizontal branch (HB) stars and red giant branch (RGB) stars in older stellar clusters [60,61] —also sets a very stringent bound of $g_{a \gamma}<0.6 \times 10^{-10} \mathrm{GeV}^{-1}$ (95\% C.L.) for $g_{a e}=0$ (for $g_{a \gamma} \sim 10^{-11} \mathrm{GeV}^{-1}$, the $95 \%$ C.L. region extends to $g_{a e} \sim 2.6 \times 10^{-13}$, as seen, for example, in the analysis of [61]) but extends to higher axion masses than the CAST bound. However, since HB and RGB stars have much higher density (by two to four orders of magnitude) and higher core temperatures (by a factor of seven) compared to the sun, mechanisms exist in the context of specific particle physics models which could allow the evasion of the bounds emerging from the null observation of axions associated with these astrophysical objects, e.g., [21-27,62-64].

The evasion could involve additional scalar degrees of freedom around the HB star temperature by invoking a phase transition [26], or the axion as a chameleon-type field with its mass depending on the environmental matter density $\rho[22,27]$. In addition, the possibility that the axion is a composite particle with a form factor has been explored
[23-25], leading to a suppression of the production in the HB stars, as well as models with a paraphoton where the axionlike particles are trapped in the HB star interior thus evading the stellar bounds.

Another possibility considers a population of axions gravitationally bound to the Sun. In [65], it is shown that stellar emission of nonrelativistic axions into gravitationally bound orbits can significantly increase the flux of axions on Earth. This additional flux reduces the coupling required to explain the XENON1T excess $\left(g_{a e} \sim 10^{-13}\right)$ and thus reduces tension with the astrophysical constraints. Further work is required to determine if this scenario can indeed provide a robust explanation of the XENON1T excess.

In Fig. 3 (top), we plot $g_{a \gamma}$ vs $g_{a e}$ where contributions from both axion-electron and axion-photon couplings are included. The red shaded regions show the XENON1T excess fit without considering inverse Primakoff while the blue shaded region utilizes inverse Primakoff. We find that the improvement in $g_{a \gamma}$ due to inverse Primakoff is quite significant for $g_{a e} \lesssim 10^{-12}$, and one can see that the transition from the $g_{a e^{-}}$-dominated signal to the $g_{a \gamma^{-}}$ dominated signal occurs around $g_{a e}=10^{-12}$ and $g_{a \gamma}=10^{-10} \mathrm{GeV}^{-1}$. In the limit of small $g_{a e}$ the inverse Primakoff channel provides flat sensitivity that is especially improved for KSVZ-type models. Constraints from white dwarf luminosity function (WDLF) place bounds on $g_{a e}<2.8 \times 10^{-13}[66]$.

If the excess is due to a background phenomenon, the current data constrain the axion parameter space. We compute this constraint by testing our signal hypothesis against the $B_{0}$ model at various exposures; in Fig. 2, we show the constraint in $g_{a \gamma}$ as a function of $m_{a}$ and we find that the constraint is already better than the CAST constraint for $m_{a}>0.04 \mathrm{eV}$. In Fig. 3 (bottom), we show the next-generation xenon (G3 Xe) constraint (with a 1 kton year exposure [67]) and find that the $2 \sigma(\sim 95 \% \mathrm{CL})$ can overcome even the HB stars constraint and start exploring the mild hint $(2.4 \sigma)$ region of stellar cooling within $1 \sigma$. Interestingly, this is only possible with the inclusion of the inverse Primakoff channel since without this channel the constraint could be worse by a few orders of magnitude. We also find that our projected sensitivity for a 1 kton year exposure at a G3 LXe experiment is competitive with future helioscope experiments. The proposed DARWIN detector would achieve a 200-ton-year exposure [68], thereby covering the current HB Stars constraint. We compare the 1 kton year projection against the projected sensitivities for IAXO + with masses $m_{a}>0.1 \mathrm{eV}$, where sensitivity begins to diminish for larger masses [69]. Additionally, future direct detection experiments with directional sensitivity would be able to use the directional information to reduce backgrounds and further increase their sensitivity to solar axions. This is especially useful in the Primakoff channel, where the axion's incoming direction is approximately preserved by the photon in the relativistic limit. 

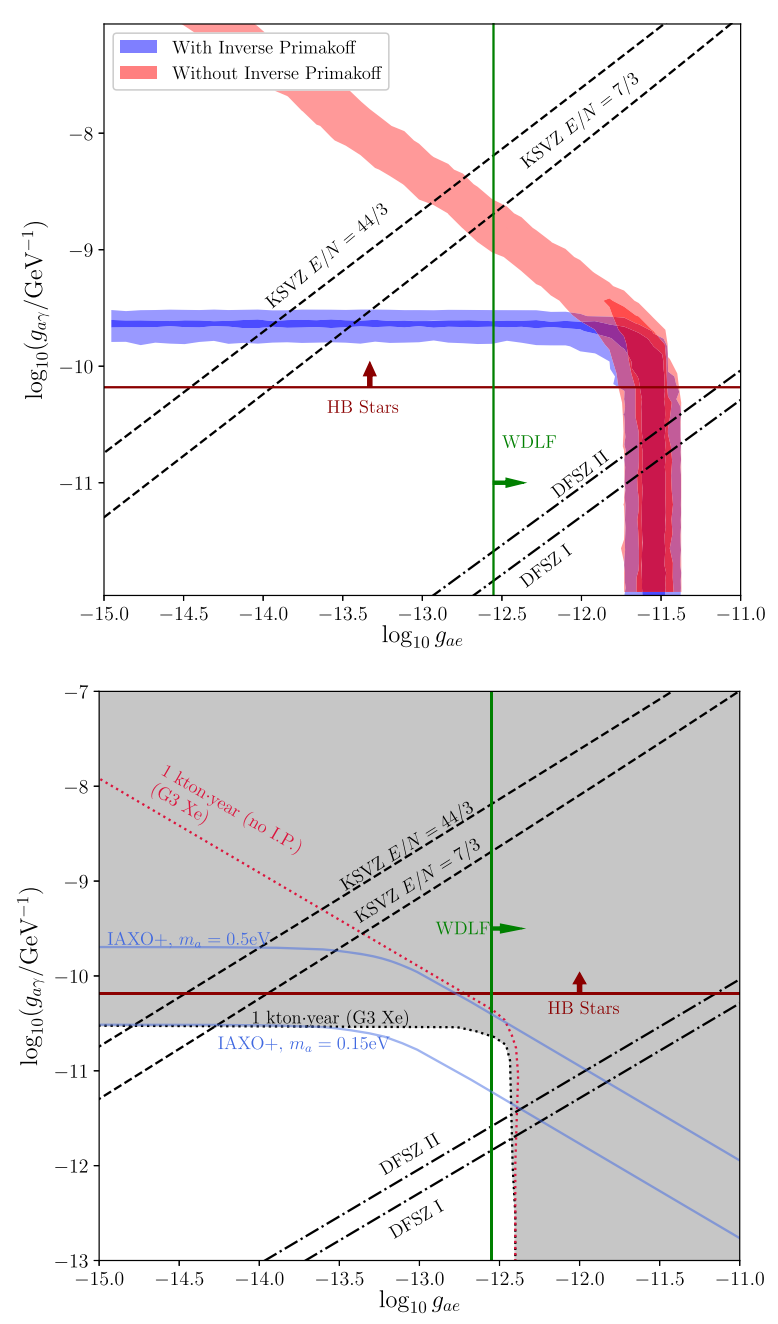

FIG. 3. Top: $2 \sigma$ credible contours are shown for fits to the XENON1T excess for all axion flux components with only axioelectric scattering (red) and with both inverse-Primakoff and axioelectric scattering (blue). $1 \sigma$ contours are also shown with dark shading. Here we consider $m_{a}=0.7 \mathrm{eV}$, however, the plot does not change for any $m_{a}<100 \mathrm{eV}$ and the CAST constraints are evaded for $m_{a}>0.03 \mathrm{eV}$. Bottom: projections of the $2 \sigma$ future exclusions (gray) set by G3 Xe over a 1 kton year exposure given background-only observations. The exclusion line for 1 kton year without inverse Primakoff (I.P.) scattering is shown for comparison (dotted red). We also show the IAXO + projection (blue) which begins to lose sensitivity for $m_{a} \gtrsim 0.01 \mathrm{eV}$.

Conclusion.-In this work, we investigated inverse Primakoff scattering as a new detection channel at liquid xenon based direct detection experiments. We showed that sole use of the coupling $g_{a \gamma}$ can fit the recent XENON1T excess. The fitting of the excess is free of the leading helioscope CAST constraint for $m_{a} \gtrsim 0.03 \mathrm{eV}$. If this excess is due to the background we find that the $95 \%$ C.L. exclusion limit is also better than the CAST limit. The tension associated with the astrophysical constraints (e.g., the WD constraint) ruling out the axion interpretation combining $g_{a e}$ and $g_{a y}$ gets relaxed with the inclusion of the inverse Primakoff effect, though the discrepancy with the $R$ parameter is still $8 \sigma$ [10]. However, these limits can be dependent on the given particle physics model. Additionally, nextgeneration xenon experiments can overcome the HB stars limit, and for $g_{a e}=10^{-13}$, the $2.4 \sigma$ hint region of stellar cooling can be probed within $1 \sigma$. In addition, these future bounds would be applicable for masses $m_{a}<1 \mathrm{keV}$, covering complementary regions of parameter space for which future helioscopes, such as IAXO, start to lose sensitivity near $m_{a} \gtrsim 0.01 \mathrm{eV}$. Further, the KSVZ model can now be probed at the direct detection experiments. A similar region of the $g_{a \gamma}-m_{a}$ space will also be investigated at LZ [70] and SuperCDMS SNOLAB [71], where the reach for $g_{a \gamma}$ needs to be scaled for the new detector type roughly by $\sqrt{M_{D} Z_{D}^{2} / M_{X e} Z_{X e}^{2}}$ (where $M_{D}$ is the detector mass and $Z_{D}$ is the atomic number of the detector nucleus) for the same exposure.

The authors thank Nicole Bell, Kiwoon Choi, and Sebastian Hoof for discussions related to this work. J. L. N. is supported in part by the Australian Research Council. J. B. D. acknowledges support from the National Science Foundation under Grant No. NSF PHY182080. B. D. and A. T. acknowledge support from DOE Grant No. DESC0010813. A. T. also thanks the Mitchell Institute for Fundamental Physics and Astronomy for support.

Note added.-Recently, a study [62] appeared that also investigated the effect of the inverse Primakoff effect on solar axion detection.

*jbdent@shsu.edu

dutta@physics.tamu.edu

*jnewstead@unimelb.edu.au

${ }^{\S}$ thompson@physics.tamu.edu

[1] D. S. Akerib et al. (LUX Collaboration), Phys. Rev. Lett. 118, 261301 (2017).

[2] C. Fu et al. (PandaX Collaboration), Phys. Rev. Lett. 119, 181806 (2017).

[3] K. Abe et al. (XMASS Collaboration), Phys. Lett. B 787, 153 (2018).

[4] E. Armengaud et al. (EDELWEISS Collaboration), Phys. Rev. D 98, 082004 (2018).

[5] E. Aprile et al. (XENON Collaboration), Phys. Rev. Lett. 123, 251801 (2019).

[6] Y. Wang et al. (CDEX Collaboration), Phys. Rev. D 101, 052003 (2020).

[7] T. Aralis et al. (SuperCDMS Collaboration), Phys. Rev. D 101, 052008 (2020).

[8] E. Aprile et al. (XENON Collaboration), arXiv:2006.09721.

[9] K. Barth et al., J. Cosmol. Astropart. Phys. 05 (2013) 010.

[10] L. Di Luzio, M. Fedele, M. Giannotti, F. Mescia, and E. Nardi, preceding Letter, Phys. Rev. Lett. 125, 131804 (2020).

[11] P. Athron et al., arXiv:2007.05517. 
[12] L. Di Luzio, M. Giannotti, E. Nardi, and L. Visinelli, arXiv:2003.01100.

[13] See Supplemental Material at http://link.aps.org/ supplemental/10.1103/PhysRevLett.125.131805 for the Inverse Primakoff Scattering diagram and more details on the spectral components of the solar axion flux.

[14] F. T. Avignone, C. Baktash, W. C. Barker, F. P. Calaprice, R. W. Dunford, W. C. Haxton, D. Kahana, R. T. Kouzes, H. S. Miley, and D. M. Moltz, Phys. Rev. D 37, 618 (1988).

[15] R. Creswick, I. Avignone, F. T., H. Farach, J. Collar, A. Gattone, S. Nussinov, and K. Zioutas, Phys. Lett. B 427, 235 (1998).

[16] R. Creswick, D. Li, F. T. Avignone, and Y. Wang, in Proceedings, 13th Patras Workshop on Axions, WIMPs and WISPs, (PATRAS 2017): Thessaloniki, Greece, 2017 (2018), pp. 11-14, http://bib-pubdb1.desy.de/record/400215.

[17] F. T. Avignone III et al. (SOLAX Collaboration), Phys. Rev. Lett. 81, 5068 (1998).

[18] M. C. D. Marsh, J. Cosmol. Astropart. Phys. 01 (2015) 017.

[19] D. Li, R. Creswick, F. Avignone, and Y. Wang, J. Cosmol. Astropart. Phys. 10 (2015) 065.

[20] J. B. Dent, B. Dutta, D. Kim, S. Liao, R. Mahapatra, K. Sinha, and A. Thompson, Phys. Rev. Lett. 124, 211804 (2020).

[21] J. Jaeckel, E. Masso, J. Redondo, A. Ringwald, and F. Takahashi, Phys. Rev. D 75, 013004 (2007).

[22] J. Khoury and A. Weltman, Phys. Rev. Lett. 93, 171104 (2004).

[23] E. Masso and J. Redondo, J. Cosmol. Astropart. Phys. 09 (2005) 015.

[24] E. Masso and J. Redondo, Phys. Rev. Lett. 97, 151802 (2006).

[25] A. Dupays, E. Masso, J. Redondo, and C. Rizzo, Phys. Rev. Lett. 98, 131802 (2007).

[26] R. N. Mohapatra and S. Nasri, Phys. Rev. Lett. 98, 050402 (2007).

[27] P. Brax, C. van de Bruck, and A.-C. Davis, Phys. Rev. Lett. 99, 121103 (2007).

[28] R. D. Peccei and H. R. Quinn, Phys. Rev. Lett. 38, 1440 (1977).

[29] S. Weinberg, Phys. Rev. Lett. 40, 223 (1978).

[30] F. Wilczek, Phys. Rev. Lett. 40, 279 (1978).

[31] M. Dine, W. Fischler, and M. Srednicki, Phys. Lett. 104B, 199 (1981).

[32] A. Zhitnitsky, Sov. J. Nucl. Phys. 31, 260 (1980).

[33] J. E. Kim, Phys. Rev. Lett. 43, 103 (1979).

[34] M. A. Shifman, A. Vainshtein, and V. I. Zakharov, Nucl. Phys. B166, 493 (1980).

[35] F. Alessandria et al. (CUORE Collaboration), J. Cosmol. Astropart. Phys. 05 (2013) 007.

[36] S. Chang and K. Choi, Phys. Lett. B 316, 51 (1993).

[37] G. G. di Cortona, E. Hardy, J. Pardo Vega, and G. Villadoro, J. High Energy Phys. 01 (2016) 034.

[38] I. G. Irastorza and J. Redondo, Prog. Part. Nucl. Phys. 102, 89 (2018).

[39] M. Giannotti, I. G. Irastorza, J. Redondo, A. Ringwald, and K. Saikawa, J. Cosmol. Astropart. Phys. 10 (2017) 010.

[40] L. Di Luzio, F. Mescia, and E. Nardi, Phys. Rev. D 96, 075003 (2017).

[41] J. Redondo, J. Cosmol. Astropart. Phys. 12 (2013) 008.

[42] S. Andriamonje et al. (CAST Collaboration), J. Cosmol. Astropart. Phys. 04 (2007) 010.
[43] C. Hagmann, H. Murayama, G. Raffelt, L. Rosenberg, and K. Bibber, Phys. Lett. B 667, 1 (2008).

[44] S. Moriyama, Phys. Rev. Lett. 75, 3222 (1995).

[45] S. Andriamonje et al. (CAST Collaboration), J. Cosmol. Astropart. Phys. 12 (2009) 002.

[46] W. C. Haxton and K. Y. Lee, Phys. Rev. Lett. 66, 2557 (1991).

[47] M. Alfonsi (XENON Collaboration), Injection and Removal of the Calibration Isotope 37ar in the Xenon1T Detector (2019), https://indico.hep.manchester.ac.uk/getFile.py/access? contribId=50\&sessionId $=4 \&$ res $I d=0 \&$ materialId $=$ slides $\&$ confId $=5456$.

[48] D. Akerib, S. Alsum, H. Arajo, X. Bai, A. Bailey, J. Balajthy, P. Beltrame, E. Bernard, A. Bernstein, T. Biesiadzinski et al., Phys. Rev. D 96, 112011 (2017).

[49] S. Dimopoulos, G. Starkman, and B. Lynn, Phys. Lett. 168B, 145 (1986).

[50] A. Ljubicic, D. Kekez, Z. Krecak, and T. Ljubicic, Phys. Lett. B 599, 143 (2004).

[51] F. T. Avignone III, R. L. Brodzinski, S. Dimopoulos, G. D. Starkman, A. K. Drukier, D. N. Spergel, G. Gelmini, and B. W. Lynn, Phys. Rev. D 35, 2752 (1987).

[52] E. Aprile et al. (XENON Collaboration), arXiv:2003.03825.

[53] E. Aprile et al. (XENON Collaboration), Phys. Rev. D 99, 112009 (2019).

[54] F. Feroz, M. Hobson, and M. Bridges, Mon. Not. R. Astron. Soc. 398, 1601 (2009).

[55] F. Feroz and M. Hobson, Mon. Not. R. Astron. Soc. 384, 449 (2008).

[56] F. Feroz, M. P. Hobson, E. Cameron, and A. N. Pettitt, Open J. Astrophys. 2, 10 (2019).

[57] V. Anastassopoulos et al. (CAST Collaboration), Nat. Phys. 13, 584 (2017).

[58] N. Vinyoles, A. Serenelli, F. L. Villante, S. Basu, J. Redondo, and J. Isern, J. Cosmol. Astropart. Phys. 10 (2015) 015.

[59] Y. Inoue, Y. Akimoto, R. Ohta, T. Mizumoto, A. Yamamoto, and M. Minowa, Phys. Lett. B 668, 93 (2008).

[60] A. Ayala, I. Dominguez, M. Giannotti, A. Mirizzi, and O. Straniero, Phys. Rev. Lett. 113, 191302 (2014).

[61] M. Giannotti, I. Irastorza, J. Redondo, and A. Ringwald, J. Cosmol. Astropart. Phys. 05 (2016) 057.

[62] C. Gao, J. Liu, L.-T. Wang, X.-P. Wang, W. Xue, and Y.-M. Zhong, following Letter, Phys. Rev. Lett. 125, 131806 (2020).

[63] I. M. Bloch, A. Caputo, R. Essig, D. Redigolo, M. Sholapurkar, and T. Volansky, arXiv:2006.14521.

[64] W. DeRocco, P.W. Graham, and S. Rajendran, arXiv:2006.15112.

[65] K. Van Tilburg, arXiv:2006.12431.

[66] M. M. Miller Bertolami, B. E. Melendez, L. G. Althaus, and J. Isern, J. Cosmol. Astropart. Phys. 10 (2014) 069.

[67] M. Szydagis (LUX and LZ Collaborations), Proc. Sci., ICHEP2016 (2016) 220 [arXiv:1611.05525].

[68] J. Aalbers et al. (DARWIN Collaboration), J. Cosmol. Astropart. Phys. 11 (2016) 017.

[69] E. Armengaud et al. (IAXO Collaboration), J. Cosmol. Astropart. Phys. 06 (2019) 047.

[70] D. Akerib et al. (LZ Collaboration), Nucl. Instrum. Methods Phys. Res., Sect. A 953, 163047 (2020).

[71] R. Agnese et al. (SuperCDMS Collaboration), Phys. Rev. D 95, 082002 (2017). 